\title{
FLORISTIC COMPOSITION AND VEGETATION STRUCTURE OF THE KNUST BOTANIC GARDEN, KUMASI, GHANA
}

\author{
A. K. Anning, S. Akyeampong, P. Addo-Fordjour, K. K. Anti, A. Kwarteng and Y. F. Tettey \\ Department of Theoretical and Applied Biology, \\ Kwame Nkrumah University of Science and Technology, Kumasi, Ghana
}

\begin{abstract}
The diversity, relative importance, canopy height and cover of plant species in the Kwame Nkrumah University of Science and Technology (KNUST) Botanic Garden were evaluated in five 1-ha plots using a stratified random sampling technique in order to build an understanding of its floristic composition and structure in two distinct parts of the garden (cultivated and uncultivated). We recorded 184 species which belonged to 146 genera, 51 families and six growth forms. The most dominant tree species in the garden were Elaeis guineensis Jacq., Hevea brasiliensis (Willd.) Muell.-Arg. and Parkia biglobosa (Jacq.) G. Don. with a combined importance index of 60.09 (20 $\%$ relative importance). The differences in importance value indices of species between the cultivated and uncultivated areas of the garden were statistically insignificant $(p>0.05)$, suggesting the presence of conducive growth environments for plants in both areas. The most dominant families were Fabaceae, Moraceae, Arecaceae and Euphorbiaceae whilst trees were the most predominant growth forms (62.5\%). Average crown height and percentage canopy were $28.8 \pm 8.81 \mathrm{~m}$ and 66.4 $\pm 8.26 \%$ respectively. These results show the floristic richness of the KNUST botanic garden and underscore the garden's potential as a centre for ex-situ conservation beside its traditional roles in education, research and recreation.
\end{abstract}

Keywords: Floristic composition, structure, botanic garden, KNUST

\section{INTRODUCTION}

Botanic gardens hold documented collections of living plants for the purposes of scientific research, conservation, aesthetic appreciation and education (Willis, 2004). According to the Botanic Garden Conservation International (BGCI, 2005), botanic gardens and arboreta together maintain over four million living plant collec- tions worldwide, most of which are deliberately collected to demonstrate their biological, ecological, taxonomic, evolutionary, conservation, ornamental, historical and cultural values. In Africa, there are only 118 (approximately $5 \%$ of the world's total) of these institutions (WyseJackson and Sutherland, 2000; Willis, 2004). Ghana has five: the Kwame Nkrumah University 
of Science and Technology (KNUST) Botanic Garden, University of Ghana Botanic Garden, University of Cape Coast Botanic Garden, Aburi Botanic Garden and the Bunso Arboretum.

The KNUST Botanic Garden is the third largest (12.9 ha) in Ghana and is also listed among the 2000 botanic gardens and arboreta of the world (Willis et al., 2002; BGCI, 2005). The garden has played significant roles in education, research and recreation since its establishment in 1960. This garden, although perceived to be floristically rich (containing tropical palms, timbers and medicinal plants as well as specialised collections of exotic plants), lacks carefully compiled and up-to-date data on the flora. This knowledge gap does not only undermine the effective functioning of the garden, but also fails to depict modern practices and trends in botanic garden management. In most modern botanic gardens, the specialized collections of plants are scientifically arranged, labelled and documented (Willis, 2004).

The rapid disappearance of genetic resources, particularly from the wild, has clearly made botanic gardens important centres for ex-situ conservation of the world's biological diversity (Smith et al., 2004). Understanding of the floristic composition and structure of botanic gardens is thus of primary importance in identifying essential elements of plant diversity, protecting threatened and economic species, monitoring the state of the garden and ultimately in the planning and implementation of biological diversity conservation (Tilman, 1988; Ssegawa and Nkuutu, 2006). A systematic floristic inventory is also essential for monitoring the spatial and temporal dynamics that may occur in the garden as a consequence of natural and anthropogenic disturbances (Bhatt et al., 1994).

This study was generally aimed at generating a comprehensive list of plant species in the KNUST Botanic Garden as well as determining the structure of the vegetation. The data gathered is expected to highlight the floristic richness of the garden and serve as baseline information for further research into strategies for the sustainable management of the garden.

\section{MATERIALS AND METHODS \\ Sampling Sites and Design}

The study was conducted in the KNUST Botanic Garden located at the heart of the KNUST campus. Two distinct areas are easily recognizable in the garden: a cultivated area, where most of the plants have been introduced deliberately to enrich the flora; and an uncultivated area which is largely without any introductions of plants and can be described as a secondary forest. Based on this, a stratified random sampling design was employed to locate five 1-ha plots (three in the cultivated and two in the uncultivated) for the study. The plots were demarcated with the help of a field compass and the edges marked with pegs and flags. Each plot was further divided into $16(25 \mathrm{~m} \times 25 \mathrm{~m})$ subplots. Sampling was done from December, 2005 to April, 2006.

\section{Floristic Composition Determination}

The sixteen subplots within each hectare plot were systematically surveyed to identify all trees (diameter at breast height, $\mathrm{dbh} \geq 10 \mathrm{~cm}$ ) and their densities determined. The basal areas of the plants were determined from their respective diameters [basal area $=\pi(\mathrm{dbh} / 2)^{2}$ ]. Densities of shrubs $(\mathrm{dbh}<10 \mathrm{~cm}$; height $>1.5 \mathrm{~m})$ were determined in $10 \mathrm{~m} \mathrm{x} 10 \mathrm{~m}$ nested plots and $1 \mathrm{~m} \mathrm{x}$ $1 \mathrm{~m}$ nested plots were used to sample herbs and seedlings $(<1.5 \mathrm{~m})$. Epiphytes and climbers were also identified and counted. Identification was done with the help of plant taxonomists. Voucher specimens were collected for plants that could not be identified in the field. These were brought to the Department of Theoretical and Applied Biology, KNUST herbarium for identification. In some cases, standard keys were used to aid in identification (Hutchinson \& Dalziel, 1963).

The Cottam and Curtis' Important Value Index (I.V.) which measures the relative importance of species (van Andel, 2003) was computed for all trees as follows: 
I. $\mathrm{V}=$ Relative density + relative frequency + relative dominance

where,

$$
\begin{aligned}
\text { Relative density } & =\frac{\text { Number of individuals of a species }}{\text { Total number of individuals }} \times 100 \\
\text { Relative frequency } & =\frac{\text { Frequency of a species }}{\text { Sum of frequencies of all species }} \times 100 \\
\text { Relative dominance } & =\frac{\text { Basal area of a species }}{\text { Total basal area of all species }} \times 100
\end{aligned}
$$

The Shannon diversity index $\left(H^{l}\right)$ and evenness (E) (Begon et al., 1996; Cox, 2002) were also calculated for the trees, shrub and herb layers of the five plots as follows:

$$
\begin{gathered}
H^{1}=-\sum_{i=1}^{s} p i \ln p_{i} \\
E=\frac{H}{\operatorname{In} S}
\end{gathered}
$$

where,

$p_{i}=$ proportion of the ith species

$\operatorname{Ln} p_{i}=$ natural log of pi

$E=$ evenness or equitability

$S=$ species richness

Determination of Canopy Cover and Height

The percentage canopy cover of each hectare plot was determined using a spherical densiometer. Four readings from the four cardinal directions were taken at four different points to obtain an average for each plot. Average canopy height was obtained by measuring the height of ten trees using a clinometer.

\section{Statistical Analysis}

Analysis of variance (ANOVA) was performed on the important value indices of species to investigate if any significant difference existed among them. The GenStat Discovery Edition 2 (VSN International Ltd, Hemel Hempstead, UK) software was used, assuming a significance level of $5 \%$.

\section{RESULTS AND DISCUSSION Floristic Composition of the KNUST Botanic Garden}

A total of 184 plant species distributed into 146 genera, 51 families and six growth forms/habits were identified within the five 1-hectare plots sampled in the garden (Table 2), a summary of which is given in Table 1. The list of species obtained is clearly not exhaustive, considering the fact that the data collected could not have been independent of the sample size (Richards, 1996) vis-à-vis the total area of the garden. It however, reflects the diverse composition of the flora in the garden. Apparently, most of the species had become established in the garden as a result of deliberate introductions and successful natural recruitments. The large number of species identified in this study, including $66.3 \%$ natives and $33.7 \%$ exotics (Hutchinson and Dalziel, 1963; Hawthorne, 1990), demonstrates that conditions prevalent in the garden are conducive to plant growth.

In general, tree species diversity was highest at the cultivated portions $\left(H^{l}=3.34 \pm 0.45\right)$ than the uncultivated portions $\left(H^{l}=2.66 \pm 0.85\right)$ of the garden (Table 1). This is attributable to the high species richness of the former and the less equitable distribution of individual trees in the latter. For instance, the pararubber plant - Hevea brasiliensis (Willd. ex Juss.) Muell.-Arg. - alone accounted for approximately $50 \%$ of all trees in the uncultivated area of the garden. Unlike the cultivated areas, the vegetation in the unculti- 
vated areas of the garden appeared to be somewhat loosely protected from anthropogenic disturbances, hence the negative impact on species diversity (Pennisi, 2005). Despite the difference in species diversity, the two areas of the garden surveyed did not differ significantly $(p>0.05)$ with respect to the important value indices of species. This indicates the lack of any strong preference for either part of the garden by the species. It also implies that both sites of the garden offer suitable environments for the growth of all forms of plants.

Table 1: Summary of the floristic composition and vegetation structure of the five one-hectare plots studied in the KNUST botanic garden

\begin{tabular}{|c|c|c|c|c|c|}
\hline \multirow{2}{*}{ Attribute } & \multicolumn{3}{|c|}{ Cultivated } & \multicolumn{2}{|c|}{ Uncultivated } \\
\hline & Plot 1 & Plot 2 & Plot 3 & Plot 4 & Plot 5 \\
\hline \multicolumn{6}{|l|}{ Tree Layer $\geq 10 \mathrm{~cm} \mathrm{dbh}$} \\
\hline Number of individuals & 161 & 175 & 163 & 203 & 153 \\
\hline $\begin{array}{l}\text { Number of species } \\
\text { Number of families }\end{array}$ & $\begin{array}{l}54^{\mathrm{a}} \\
22^{\mathrm{a}}\end{array}$ & $\begin{array}{l}35^{\mathrm{a}} \\
21^{\mathrm{a}}\end{array}$ & $\begin{array}{l}63^{\mathrm{a}} \\
32^{\mathrm{a}}\end{array}$ & $\begin{array}{l}57^{\mathrm{a}} \\
23^{\mathrm{a}}\end{array}$ & $\begin{array}{l}31^{\mathrm{a}} \\
18^{\mathrm{a}}\end{array}$ \\
\hline Mean diameter of trees $(\mathrm{cm})$ & 85.1 & 90 & 68 & 89.5 & 110 \\
\hline Shannon diversity index $\left(H^{l}\right)$ & 3.55 & 2.83 & 3.65 & 3.25 & 2.07 \\
\hline Shannon evenness $(E)$ & 0.88 & 0.80 & 0.89 & 0.79 & 0.60 \\
\hline Mean canopy height (m) & $44 \pm 36$ & $23 \pm 15$ & $29 \pm 22$ & $24 \pm 15$ & $24 \pm 15$ \\
\hline Mean canopy cover $(\%)$ & $70 \pm 9$ & $52 \pm 15$ & $68 \pm 13$ & $69 \pm 9$ & $73 \pm 3$ \\
\hline \multicolumn{6}{|c|}{ Shrub Layer $<10 \mathrm{~cm} \mathrm{dbh}$ and height $\geq 1.5 \mathrm{~m}$} \\
\hline Number of individuals & 27 & 0 & 87 & 28 & 84 \\
\hline Number of species & $11^{\mathrm{a}}$ & $0^{\mathrm{a}}$ & $22^{\mathrm{a}}$ & $20^{\mathrm{a}}$ & $24^{\mathrm{a}}$ \\
\hline Number of families & $10^{\mathrm{a}}$ & $0^{\mathrm{a}}$ & $13^{\mathrm{a}}$ & $17^{\mathrm{a}}$ & $15^{\mathrm{a}}$ \\
\hline Shannon diversity index $\left(H^{l}\right)$ & 2.03 & 0 & 2.64 & 2.87 & 2.20 \\
\hline Shannon evenness $(E)$ & 0.85 & 0 & 0.85 & 0.96 & 0.69 \\
\hline \multicolumn{6}{|l|}{ Herb Layer $<1.5 \mathrm{~m}$} \\
\hline Number of individuals & 183 & 158 & 74 & 74 & 47 \\
\hline Number of species & $21^{\mathrm{a}}$ & $22^{\mathrm{a}}$ & $15^{\mathrm{a}}$ & $17^{\mathrm{a}}$ & $5^{\mathrm{a}}$ \\
\hline Number of families & $19^{\mathrm{a}}$ & $15^{\mathrm{a}}$ & $13^{\mathrm{a}}$ & $13^{\mathrm{a}}$ & $5^{\mathrm{a}}$ \\
\hline Shannon diversity index $\left(H^{l}\right)$ & 2.39 & 2.66 & 1.96 & 0.30 & 1.41 \\
\hline Shannon evenness $(E)$ & 0.79 & 0.86 & 0.72 & 0.11 & 0.88 \\
\hline
\end{tabular}

${ }^{a}$ the numbers do not add up to the total number of species and families identified in the study because of overlaps across the two portions of the garden sampled.

106 Journal of Science and Technology, Vol. 28, No. 3, Dec., 2008 
Table 2: List of plant species identified in the KNUST botanic garden with their families, growth habits and important value indices (IVs)

\begin{tabular}{|c|c|c|c|}
\hline Species & Family & Habit & IV \\
\hline Acoelorrhape wrightii (Griseb \& Wendl.) Britoon ${ }^{a}$ & Arecaceae & Tree & 0.22 \\
\hline Acridocarpus natalitius A. Juss. & Malpighiaceae & Climber & - \\
\hline Afzelia africana Sm. ex Pers. & Fabaceae & Tree & 1.09 \\
\hline Ageratum conyzoides $\mathrm{L}^{\mathrm{a}}$ & Asteraceae & Herb & - \\
\hline Albizia adianthifolia (Schum.) W. F. Wight & Fabaceae & Tree & 3.24 \\
\hline Albizia ferrugenia (Guill. \& Perr.) Benth. & Fabaceae & Tree & 6.10 \\
\hline Albizia zygia (DC.) J. F. Macbr. & Fabaceae & Tree & 4.38 \\
\hline Alchornia cordifolia (Schum. \& Thonn.) Muel-Arg & Euphorbiaceae & Shrub & - \\
\hline Allanblackia floribunda A. Chev. & Clusiaceae & Tree & 1.32 \\
\hline Aloe macrocarpa Tod. ${ }^{\mathrm{a}}$ & Aloaceae & Herb & - \\
\hline Alstonia boonei de Wild & Apocynaceae & Tree & 4.02 \\
\hline Amphimas pterocarpoides Harms & Fabaceae & Tree & 3.01 \\
\hline Anacardium occidentale L. & Anacardiaceae & Tree & 0.32 \\
\hline Anthocleista nobilis G. Don & Loganiaceae & Tree & 2.16 \\
\hline Anthocleista vogelii Planch. & Loganiaceae & Tree & 0.33 \\
\hline Anthonota macrophylla P. Beauv. & Fabaceae & Tree & 2.20 \\
\hline Antiaris toxicaria (Pers.) Lesch. & Moraceae & Tree & 9.19 \\
\hline Artocarpus nobilis Thwaites & Moraceae & Tree & 1.71 \\
\hline Aspilia africana (Pers.) C. A. Adams & Asteraceae & Herb & - \\
\hline Aubrevillea kerstingii (Harms) Pellegrin & Fabaceae & Tree & 0.97 \\
\hline Axonopus compressus (Sw.) Beauv. & Poaceae & Grass & - \\
\hline Bambusa vulgaris Schrad. ex Wendl. ${ }^{\text {a }}$ & Poaceae & $\begin{array}{l}\text { Tree-like } \\
\text { grass }\end{array}$ & - \\
\hline Baphia nitida Lodd & Fabaceae & Shrub & - \\
\hline Blighia sapida Koenig & Sapindaceae & Tree & 1.44 \\
\hline Blighia unijugata Baker & Sapindaceae & Tree & 0.86 \\
\hline Bombax buonopozense Beauv. & Bombacaceae & Tree & 7.43 \\
\hline Bridelia atroviridis Muell.-Arg. & Euphorbiaceae & Tree & 0.91 \\
\hline Bridelia $\mathrm{sp}$ & Euphorbiaceae & Tree & 0.37 \\
\hline Calathea cyclophora Baker $^{\mathrm{a}}$ & Maranthaceae & Herb & - \\
\hline Canthium hispidum Benth. & Rubiaceae & Climber & - \\
\hline Carapa procera DC & Meliaceae & Tree & 3.46 \\
\hline Caryota mitis Lour. $^{\text {a }}$ & Arecaceae & Tree & 0.25 \\
\hline Cassia nodosa Roxb & Fabaceae & Tree & 0.74 \\
\hline Casuarina equisitifolia Forster \& Forster.f. ${ }^{\text {a }}$ & Casuarinaceae & Tree & 3.41 \\
\hline Cedrela odorata $\mathrm{L}^{\mathrm{a}}$ & Meliaceae & Tree & 0.37 \\
\hline Ceiba pentandra (L.) Gaertn & Bombacaceae & Tree & 6.71 \\
\hline
\end{tabular}


Floristic composition and vegetation structure of the KNUST ...

Anning et al.

\begin{tabular}{|c|c|c|c|}
\hline Species & Family & Habit & IV \\
\hline Centrosema pubescens Benth. $^{a}$ & Fabaceae & Herb & - \\
\hline Chromolaena odorata (L.) King \& Robin. ${ }^{a}$ & Asteraceae & Shrub & - \\
\hline Chrysopogon acicularis (Retz.) Trin. ${ }^{a}$ & Poaceae & Grass & - \\
\hline Cinnamomum zeylandicum Breyn. ${ }^{\text {a }}$ & Lauraceae & Tree & 0.42 \\
\hline Citrus aurantifolia (Christm. ) Swingle ${ }^{a}$ & Rutaceae & Tree & 0.48 \\
\hline Citrus nobilis Lour. $^{\mathrm{a}}$ & Rutaceae & Tree & 0.65 \\
\hline Citrus sinensis (L.) Osbeck ${ }^{\mathrm{a}}$ & Rutaceae & Tree & 0.82 \\
\hline Cnestis ferruginea DC. & Connaraceae & Shrub & - \\
\hline Cola gigantea A. Chev. & Sterculiaceae & Tree & 4.97 \\
\hline Cola reticulata A. Chev & Sterculiaceae & Tree & 1.83 \\
\hline Cola sp. & Sterculiaceae & Tree & 2.63 \\
\hline Colocasia esculenta (L.) Schott. ${ }^{\text {a }}$ & Araceae & Herb & - \\
\hline Combretum hispidum C. Lawson ${ }^{\mathrm{a}}$ & Combretaceae & Climber & - \\
\hline Combretum sp. & Combretaceae & Tree & 1.84 \\
\hline Commelina erecta $\mathrm{L}^{\mathrm{a}}$ & Commelinaceae & Herb & - \\
\hline Commelina latifolia C. B. Clarke ${ }^{a}$ & Commelinaceae & Herb & - \\
\hline Culcacia angolensis Welw. ex. Schott. & Araceae & Herb & - \\
\hline Cyanthillium cinereum (L.) H. E. Robins. ${ }^{a}$ & Asteraceae & Herb & - \\
\hline Cyperus rotundus $\mathrm{L}^{\mathrm{a}}$ & Cyperaceae & Sedge & - \\
\hline Dalbergia hostilis Benth. & Fabaceae & Climber & - \\
\hline Dalbergia sexatilis Hooker $\mathrm{f}$. & Fabaceae & Climber & - \\
\hline Daniellia ogea (Harms) Rolfe ex Holland & Fabaceae & Tree & 0.37 \\
\hline Desmodium adscendens (Sw.) DC. ${ }^{\mathrm{a}}$ & Fabaceae & Herb & - \\
\hline Dialum guinense Willd. & Fabaceae & Tree & 1.76 \\
\hline Diospyros mespiliformis Hochst. ex A. DC. ${ }^{a}$ & Ebenaceae & Tree & 1.11 \\
\hline Dioscorea alata L. ${ }^{\mathrm{a}}$ & Dioscoreaceae & Climber & - \\
\hline Dioscorea bulbifera Linne & Dioscoreaceae & Climber & - \\
\hline Dioscorea preusii $\mathrm{Pax}$ & Dioscoreaceae & Climber & - \\
\hline Dioscorea smilacifolia Wildem and Durand. & Dioscoreaceae & Climber & - \\
\hline Dissotis rotundifolia $(\mathrm{Sm}$.$) Triana ^{\mathrm{a}}$ & Melastomataceae & Herb & - \\
\hline Distemonanthus benthamianus Baillon & Fabaceae & Tree & 1.73 \\
\hline Dracaena arborea (Willd.) Link & Dracaenaceae & Tree & 0.32 \\
\hline Dracaena mannii Baker & Dracaenaceae & Tree & 0.33 \\
\hline Drypetes sp. Vahl. ${ }^{\mathrm{a}}$ & Euphorbiaceae & Herb & - \\
\hline Duranta erecta L. & Verbenaceae & Shrub & - \\
\hline Echinocereus sp. ${ }^{\mathrm{a}}$ & Cactaceae & Tree & 1.10 \\
\hline
\end{tabular}

108 Journal of Science and Technology, Vol. 28, No. 3, Dec., 2008 
Floristic composition and vegetation structure of the KNUST ...

Anning et al.

\begin{tabular}{|c|c|c|c|}
\hline Species & Family & Habit & IV \\
\hline Elaeis guineensis Jacq. & Arecaceae & Tree & 34.68 \\
\hline Emilia sanchifolia (L.) DC. & Asteraceae & Herb & - \\
\hline Entandrophragma angolense (Welw.) D.C & Meliaceae & Tree & 0.90 \\
\hline Entandrophragma candollei Harms. & Meliaceae & Tree & 1.31 \\
\hline Euadenia trifoliolata (Schum. \& Thonn.) Oliv & Capparidaceae & Tree & 0.33 \\
\hline Eugenia longiflora (K. Presl.) Fer.- Vill. & Myrtaceae & Tree & 0.33 \\
\hline Fadherbia albida (Del.) A. Chev. ${ }^{a}$ & Fabaceae & Tree & 0.87 \\
\hline Ficus barteri Sprague & Moraceae & Tree & 0.51 \\
\hline Ficus exasperata Vahl. & Moraceae & Tree & 3.24 \\
\hline Ficus ottoniifolia (Miq.) Miq. & Moraceae & Epiphyte & - \\
\hline Ficus ovata Vahl. & Moraceae & Epiphyte & - \\
\hline Ficus polita Vahl. & Moraceae & Epiphyte & - \\
\hline Ficus saussureana DC & Moraceae & Tree & 0.26 \\
\hline Ficus tessellata Warb. & Moraceae & Epiphyte & - \\
\hline Ficus thonningii Blume & Moraceae & Epiphyte & - \\
\hline Ficus trichopoda Baker & Moraceae & Epiphyte & - \\
\hline Ficus umbellata Vahl & Moraceae & Epiphyte & - \\
\hline Ficus vogelii (Miq.) Miq & Moraceae & Epiphyte & - \\
\hline Ficus vogelii var. pubicarpa & Moraceae & Epiphyte & - \\
\hline Funtumia elastica (Preuss) Stapf. & Apocynaceae & Tree & 1.06 \\
\hline Garcinia gnetoides Hutch. \& Dalz. ${ }^{a}$ & Myrtaceae & Tree & 3.28 \\
\hline Griffonia simplicifolia (Vahl ex DC) Baillon & Fabaceae & Shrub & - \\
\hline Hevea brasiliensis (Willd. ex Juss.) Muell.-Arg ${ }^{a}$ & Euphorbiaceae & Tree & 14.52 \\
\hline Hippocratea africana (Wild.) Loesener ex Engler & Celastraceae & Climber & - \\
\hline Hippocratea macrophylla Vahl. & Celastraceae & Climber & - \\
\hline Holarrhena floribunda (G. Don) Durand \& Schinz. & Apocynaceae & Tree & 2.43 \\
\hline Howea forsteriana Becc. $^{\mathrm{a}}$ & Arecaceae & Tree & 1.50 \\
\hline Ipomoea involucrata $\mathrm{P} . \mathrm{Beauv}^{\mathrm{a}}$ & Convolvulaceae & Climber & - \\
\hline Justicia flava Vahl. $^{\mathrm{a}}$ & Acanthaceae & Herb & - \\
\hline Lannea welwitschii (Hiern) Engl. & Anacardiaceae & Tree & 1.62 \\
\hline Leucaena leucocephala (Lam.) de Wit. ${ }^{a}$ & Fabaceae & Tree & 3.22 \\
\hline Licuala peltata.( Roxb. ex Buch) Ham ${ }^{\mathrm{a}}$ & Arecaceae & Tree & 1.18 \\
\hline Livistonia sp. ${ }^{\mathrm{a}}$ & Arecaceae & Tree & 0.72 \\
\hline Lophira alata Banks ex Gaertn.f. & Ochnaceae & Tree & 1.00 \\
\hline
\end{tabular}


Floristic composition and vegetation structure of the KNUST ...

Anning et al.

\begin{tabular}{|c|c|c|c|}
\hline Species & Family & Habit & IV \\
\hline Macaranga hurifolia Beille. & Euphorbiaceae & Tree & 0.42 \\
\hline Mallotus oppositifolius (Geiseler) Muell-Arg ${ }^{\mathrm{a}}$ & Euphorbiaceae & Shrub & - \\
\hline Mangifera indica L. $^{\text {a }}$ & Anacardiaceae & Tree & 5.54 \\
\hline Mansonia altissima A. Chev. & Sterculiaceae & Tree & 0.42 \\
\hline Mareya micrantha (Benth.) Muell-Arg. & Euphorbiaceae & Tree & 1.55 \\
\hline Milicia excels (Welw.) C. C. Berg. & Moraceae & Tree & 1.10 \\
\hline Millittia rhodantha Baillon & Fabaceae & Tree & 0.31 \\
\hline Mitragyna stipulosa (DC) O. Kuntze ${ }^{\mathrm{a}}$ & Rubiaceae & Tree & 0.40 \\
\hline Momordica angustisepala Harms. & Cucurbitaceae & Climber & - \\
\hline Morinda lucida Benth. & Rubiaceae & Tree & 7.59 \\
\hline Morus mesozygia Stapf. & Moraceae & Tree & 2.32 \\
\hline Motandra guinensis (Thonn.) DC & Apocynaceae & Climber & - \\
\hline Myrianthus arboreus P. Beauv. & Moraceae & Tree & 1.97 \\
\hline Myrianthus libericus Rendle & Moraceae & Tree & 0.28 \\
\hline Nephrolepis biserrata (Sw.) Schott ${ }^{\mathrm{a}}$ & Nephrolepidaceae & Herb (fern) & - \\
\hline Nesogordonia papaverifera (A. Chev.) R Capuron & Sterculiaceae & Tree & 0.43 \\
\hline Oplismenus burmanii (Retz.) Beauv. & Poaceae & Grass & - \\
\hline Oxalis corniculata $\mathrm{L}$. & Oxalidaceae & Herb & - \\
\hline Parkia biglobosa (Jacq.) R. Br. ex G. Don. & Fabaceae & Tree & 10.89 \\
\hline Parkia filicoidea Welw. ex Oliver & Fabaceae & Tree & 1.49 \\
\hline Parquetina nigrescens (Afzel.) Bullock. & Periplocaceae & Climber & - \\
\hline Paspalum notatum Flugge ${ }^{\mathrm{a}}$ & Poaceae & Grass & - \\
\hline Peltophoreum pterocarpum (DC.) Backer ex Heyne ${ }^{a}$ & Fabaceae & Tree & 5.63 \\
\hline Pereskia bleo (Kunth) DC ${ }^{\mathrm{a}}$ & Cactaceae & Tree & 0.47 \\
\hline Phoenix dactylifera L. ${ }^{\mathrm{a}}$ & Arecaceae & Tree & 1.55 \\
\hline Phoenix reclinata Jacq. ${ }^{\mathrm{a}}$ & Arecaceae & Tree & 2.30 \\
\hline Phyllanthus amarus Schum. \& Thonn. & Euphorbiaceae & Herb & - \\
\hline Phyllanthus discoideus (Baill.) Muell.-Arg. & Euphorbiaceae & Herb & - \\
\hline Pinus caribaea Morelet. $^{\mathrm{a}}$ & Pinaceae & Tree & 1.17 \\
\hline Piper guineense Schum. and Thonn. & Piperaceae & Climber & - \\
\hline Piptadeniastrum africanum (Hook. f.) Brenan & Fabaceae & Tree & 3.36 \\
\hline Pritchardia affinis Becc. ${ }^{\text {a }}$ & Arecaceae & Tree & 1.00 \\
\hline Pseudospondias microcarpa (A. Rich) Engl. & Anacardiaceae & Tree & 8.75 \\
\hline Psidium guajava $\mathrm{L}^{\mathrm{a}}$ & Myrtaceae & Tree & 0.37 \\
\hline
\end{tabular}

110 Journal of Science and Technology, Vol. 28, No. 3, Dec., 2008 
Floristic composition and vegetation structure of the KNUST ...

Anning et al.

\begin{tabular}{|c|c|c|c|}
\hline Species & Family & Habit & IV \\
\hline Psidium littorale Raddi $^{\mathrm{a}}$ & Myrtaceae & Tree & 1.22 \\
\hline Psydrax subcordata (DC) Bridson & Rubiaceae & Tree & 3.51 \\
\hline $\begin{array}{l}\text { Ptychosperma macarthirii (H. Wedl. ex Veitch) H. } \\
\text { Wedl. ex Hook. f. }\end{array}$ & Arecaceae & Tree & 0.10 \\
\hline Pycnanthus angolensis (Welw.) Warb. & Myristicaceae & Tree & 0.88 \\
\hline Raphia hookeri L. & Arecaceae & Tree & 6.06 \\
\hline Raphidosphora africana $\mathrm{N}$. E. Br. & Araceae & Climber & - \\
\hline Rauvolfia vomitoria Afzelius & Apocynaceae & Tree & 2.09 \\
\hline Reissantis indica (Willd.) Halle & Celastraceae & Climber & - \\
\hline Ricinodendron heudelotii (Baill.) Heckel & Euphorbiaceae & Tree & 2.35 \\
\hline Rothmannia longiflora Salisb. & Rubiaceae & Tree & 2.07 \\
\hline Roystonea regia (Kunth.) Cook ${ }^{\mathrm{a}}$ & Arecaceae & Tree & 1.40 \\
\hline Sabal palmetto (Walt.) Lodd. ${ }^{\mathrm{a}}$ & Arecaceae & Tree & 2.02 \\
\hline Salacia alata De. Wild. & Celastraceae & Climber & - \\
\hline Salacia reticulata Wight $^{\mathrm{a}}$ & Celastraceae & Shrub & - \\
\hline Salix babylonica $\mathrm{L}$. & Saliaceae & Shrub & - \\
\hline Scleria naumaniana Boeck. & Cyperaceae & Sedge & - \\
\hline Solanum erianthum D. Don & Solanaceae & Shrub & - \\
\hline Spathodea campanulata Beauv. & Bignoniaceae & Tree & 1.46 \\
\hline Spondias mombin L. & Anacardiaceae & Tree & 0.72 \\
\hline Sporobollus pyramidalis Beauv. ${ }^{\text {a }}$ & Poaceae & Grass & - \\
\hline Sterculia rhinopetala $\mathrm{K}$. Schum & Sterculiaceae & Tree & 0.57 \\
\hline Sterculia tragacantha Lindley & Sterculiaceae & Tree & 3.37 \\
\hline Strophanthus barteri Franch. & Apocynaceae & Climber & - \\
\hline Strychnos nux-vomica L. ${ }^{\mathrm{a}}$ & Loganiaceae & Tree & 1.32 \\
\hline Synedrella nodiflora (L.) Gaertn. & Asteraceae & Herb & - \\
\hline Tabebuia chrysantha G. Nicholson ${ }^{\mathrm{a}}$ & Bignoniaceae & Tree & 6.76 \\
\hline Tacca sp. ${ }^{\mathrm{a}}$ & Taccaceae & Herb & - \\
\hline Tamarindus indica L. ${ }^{\mathrm{a}}$ & Fabaceae & Tree & 0.82 \\
\hline Terminalia ivorensis A. Chev. & Combretaceae & Tree & 4.10 \\
\hline Terminalia superba Engl. \& Diels. & Combretaceae & Tree & 1.14 \\
\hline Tetrapleura tetraptera (Schum. \& Thonn.) Taubert & Fabaceae & Tree & 0.53 \\
\hline $\begin{array}{l}\text { Tetrorchidium didymostemon (Baill.) Pax \& K. } \\
\text { Hoffm }\end{array}$ & Euphorbiaceae & Tree & 0.69 \\
\hline Theobroma cacao $\mathrm{L}^{\mathrm{a}}$ & Sterculiaceae & Tree & 1.14 \\
\hline Trema orientalis $(\mathrm{L}$.$) Blume$ & Ulmaceae & Tree & 0.71 \\
\hline Trichilia prieuriana A. Juss. & Meliaceae & Tree & 2.47 \\
\hline Trichilia monadelpha (Thonn.) de Wild. & Meliaceae & Tree & 0.49 \\
\hline
\end{tabular}

Journal of Science and Technology, Vol. 28, No. 3, Dec., 2008111 
Floristic composition and vegetation structure of the KNUST ...

Anning et al.

\begin{tabular}{|c|c|c|c|}
\hline Species & Family & Habit & IV \\
\hline Triplochiton scleroxylon Schum. & Sterculiaceae & Tree & 0.98 \\
\hline Urochloa maxima (Jacq.) R. Webster & Poaceae & Grass & - \\
\hline Veitchia arecina Becc. ${ }^{a}$ & Arecaceae & Tree & 0.50 \\
\hline Vitex trifolia $\mathrm{L}$. & Verbenaceae & Tree & 0.39 \\
\hline Voacanga africana Stapf. & Apocynaceae & Tree & 0.97 \\
\hline Wallichia densiflora Mart. $^{\mathrm{a}}$ & Arecaceae & Tree & 1.06 \\
\hline Xylopia aethiopica (Dunal.) A Rich. & Annonaceae & Tree & 4.29 \\
\hline Zanthoxylum xantholoides Waterm & Rutaceae & Tree & 3.00 \\
\hline
\end{tabular}

${ }^{a}$ Exotic species

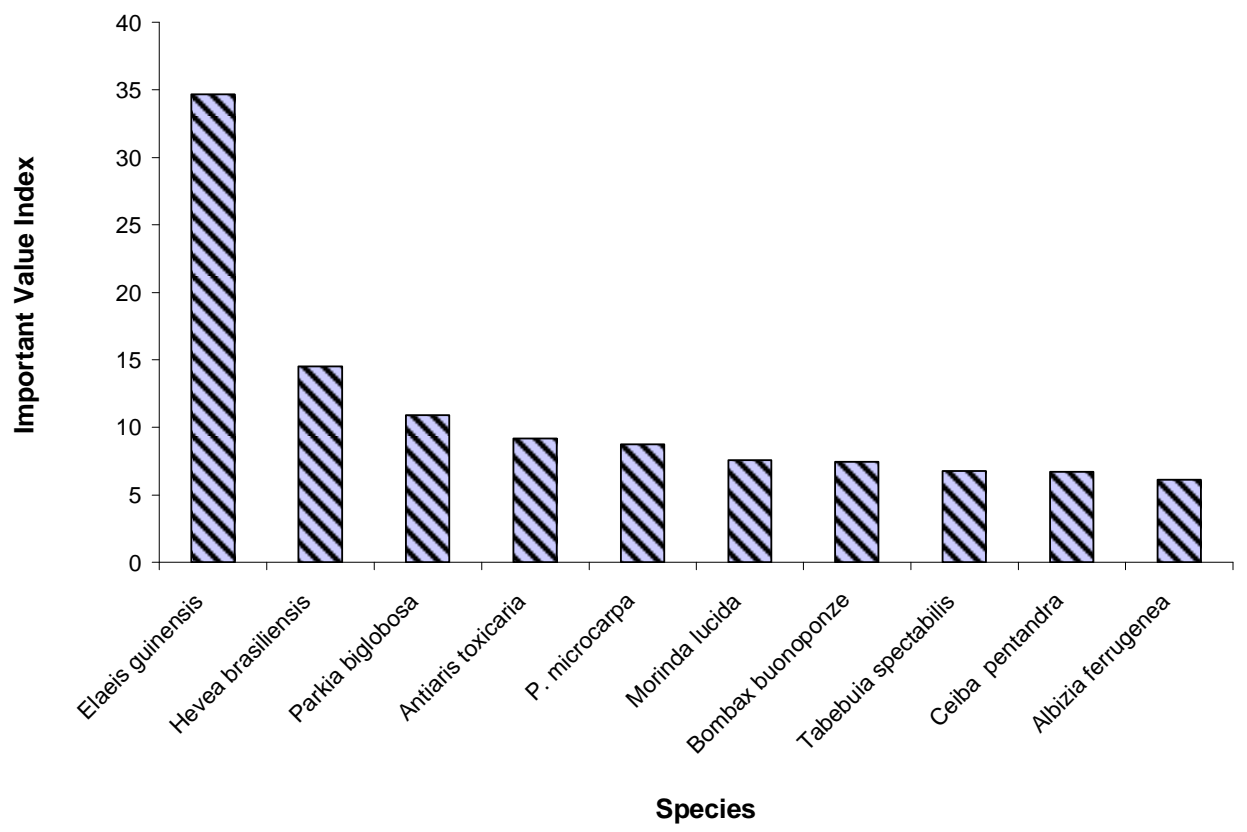

Fig. 1: Relative importance of the most dominant trees identified in the KNUST botanic garden

The important value index (IV) is a measure of the relative importance of a species in an area and combines such attributes as relative density, relative frequency and relative dominance (van Andel, 2003). Based on this, Elaeis guineensis Jacq. emerged as the most important species, recording an index value of 34.68. This was fol- lowed by $H$. brasiliensis and Parkia biglobosa (Jacq.) R. Br. Ex G. Don with IVs of 14.52 and 10.89 respectively (Fig. 1). The remaining species had IVs below 10. The important value indices of the species differed significantly $(\mathrm{df}=$ $117, \mathrm{p}<0.001)$. This supports the observation that some species were more dominant in the

112 Journal of Science and Technology, Vol. 28, No. 3, Dec., 2008 
garden than others. The three most important species, which together had approximately $20 \%$ dominance, are all economically important species and might have been intentionally introduced into the garden or received a little more attention than other species. Besides, E. guineensis and $P$. biglobosa are native plants, implying that they can thrive in most environments in the tropics. H. brasiliensis formed a monoculture of about $50 \mathrm{~m}^{2}$ area in the garden.

Fabaceae was the most species-rich family (24 species), followed by Moraceae, Arecaceae (Palmae) and Euphorbiaceae with 18, 15 and 12 species respectively. The 47 remaining families together had a total of 115 species $(62.5 \%$ of species identified in the garden), with as many as 25 families recording single species each (Table 2, Fig. 2). With respect to the number of individuals, however, the importance or dominance was highest in the Arecaceae, followed by
Euphorbiaceae, Fabaceae and Moraceae, in decreasing order (Fig. 2). The dominance of Arecaceae (Palmae) is largely attributable to the wide distribution of E. guineensis in the garden. On the other hand, the epiphytic habit of most of the species of Moraceae, and their preference for specific host trees (Munoz et al., 2003), may explain the fewer number of individuals recorded for this family. It is noteworthy that, apart from being naturally large (Langenheim and Thimann, 1982; Watson and Dallwitz, 2002), most of the species in these families (except those of Arecaceae) are native tropical plants which can thrive under tropical conditions.

The species identified in the botanic garden naturally fell into six growth forms or habits namely trees, broadleaf herbaceous plants, shrubs, epiphytes, grasses/sedges and climbers/ lianas. The trees were the most diverse growth

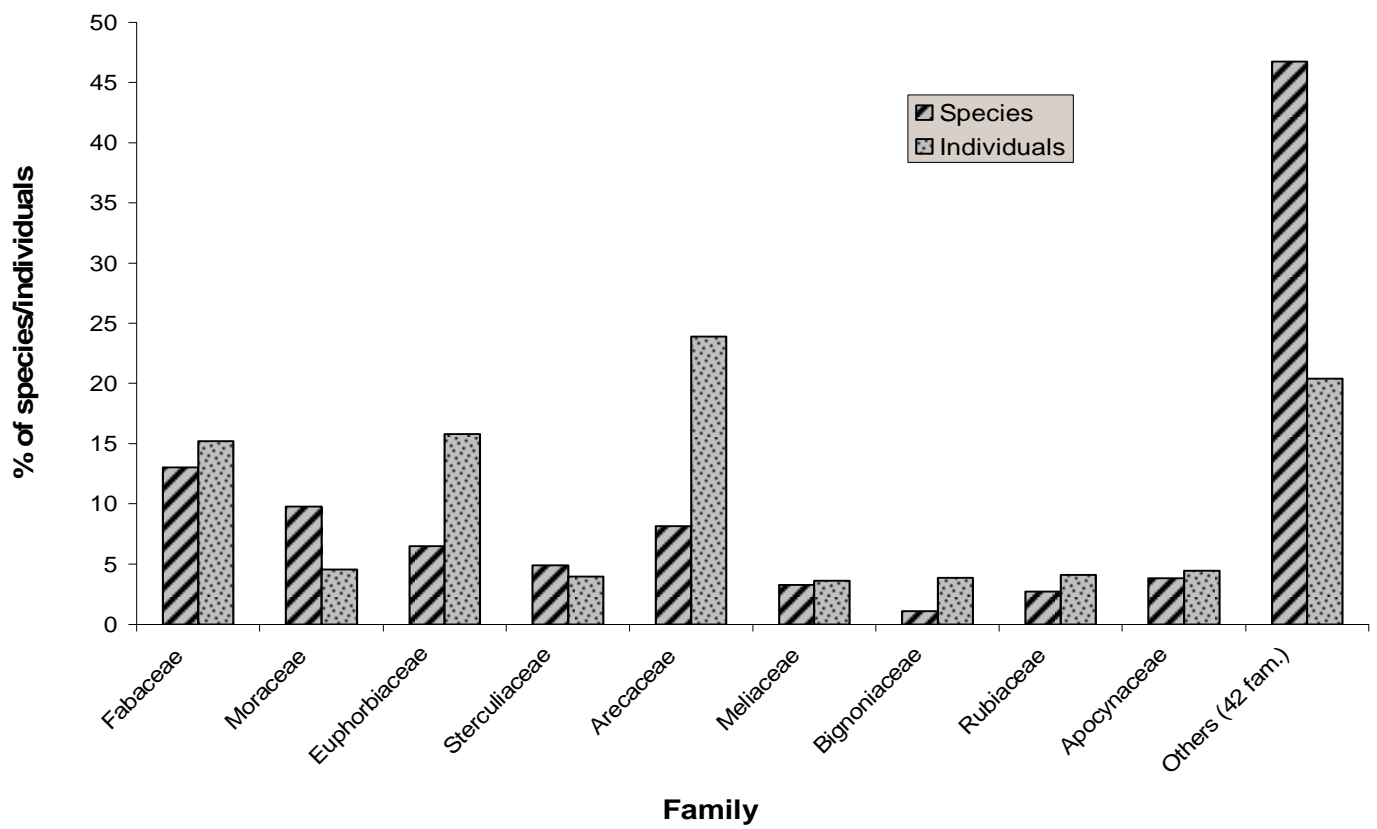

Fig. 2: Family dominance of flora in the KNUST botanic garden based on percentages of individuals and species of trees 
form, accounting for $62.5 \%$ of all species encountered in the garden, whilst epiphytes and grasses/sedges were the least common (Fig. 3). The ground layer of the cultivated part of the garden is regularly mowed to create opportunities for recreation, retreats and other end-uses. This practice appeared to have had a negative impact on the shrub composition of the garden as observed in portions of the cultivated area where no shrub was recorded (Table 1). On the contrary, availability of light, resulting mainly from the sparse spatial distribution of trees (Riffell and Gutzwiller, 1996; Pabst and Spies, 1998) coupled with the increasing accessibility to humans, appeared to have impacted positively on the herb diversity in the cultivated areas.

\section{Vegetation Structure of the Garden}

The diameter (basal area), height and percentage cover of trees in the KNUST Botanic Garden were measured to emphasize their importance in determining the garden's vegetation structure. The mean values of all three attributes were high (Table 1), suggesting the predominance of mature closed forest vegetation in the garden. Mean canopy cover and diameter of trees in the uncultivated part of the garden $(71 \%$ and $99.75 \mathrm{~cm}$ respectively) were higher compared to the cultivated, again indicating the relatively less anthropogenic interventions in the former part of the garden (Table 1). Canopy height was however higher in most parts of the cultivated area (23 $44 \mathrm{~m})$ than the uncultivated area $(24 \mathrm{~m})$. Some emergents, including Ricinodendron heudelotti (Baill.) Pierre ex Pax. and Entandrophragma candollei Kosipo, were common, again indicating the mature nature of the vegetation.

\section{CONCLUSION}

The study produced a comprehensive but incomplete list of plant species including medicinal plants, timbers, edibles/spices and other com-

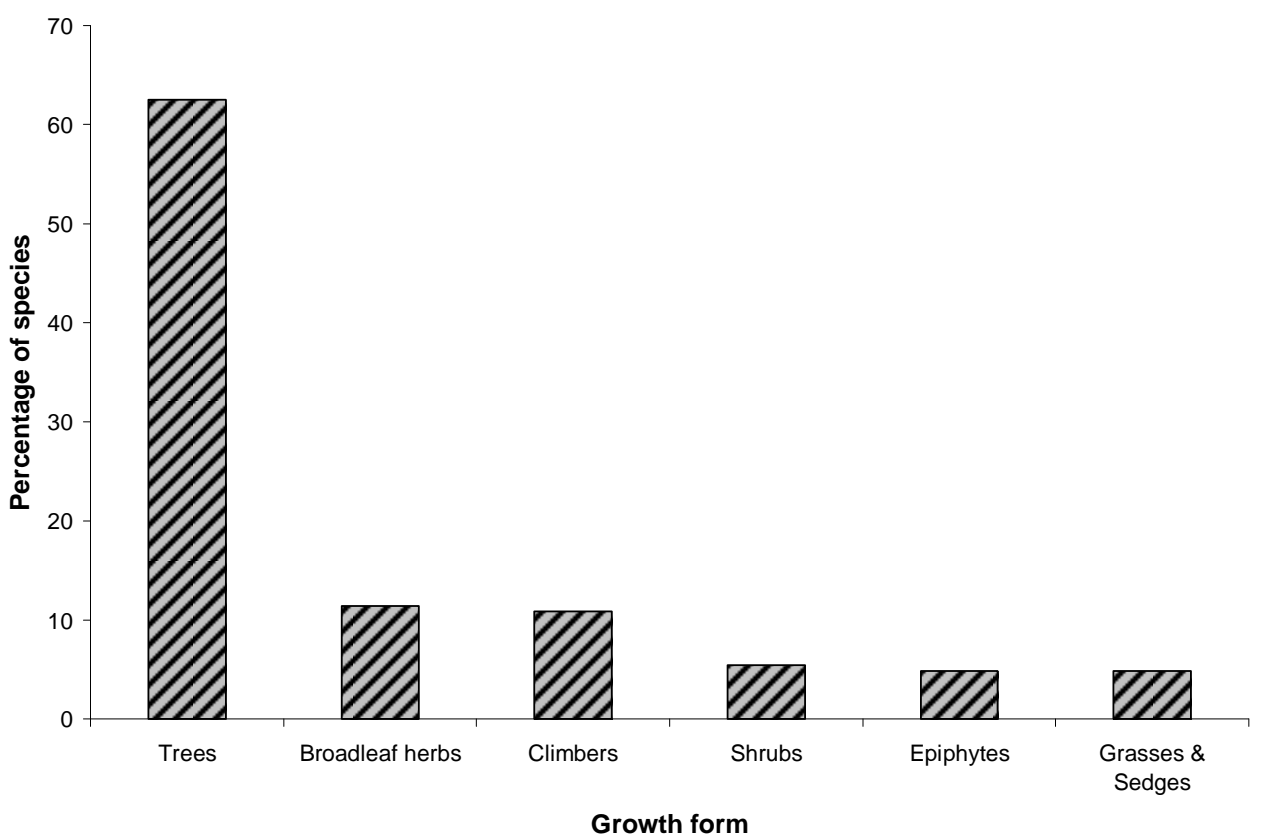

Fig. 3: Growth forms of plant species encountered in the study area

114 Journal of Science and Technology, Vol. 28, No. 3, Dec., 2008 
mercial plants. This will serve as a good reference list for future studies aimed at understanding or monitoring the spatio-temporal dynamics of vegetation in the KNUST botanic garden. It will also be a good baseline data for the development of a plant resource database for the garden. Further, with their important value indices determined, it will be easier to prioritize the species for conservation or management. The high numbers of both native and exotic plant species recorded in this study sufficiently demonstrates the garden's potential as an ex-situ conservation centre besides its traditional roles. In this regard, propagules of species locally extinct or threatened from some habitats could be obtained from the garden for reintroduction into the wild and vice versa due to the prevailing equable growth conditions.

\section{ACKNOWLEDGEMENTS}

We are grateful to the staff of the Department of Theoretical and Applied Biology, Kwame Nkrumah University of Science and Technology (KNUST), Kumasi, Ghana, for granting us access to the garden. We also acknowledge with thanks the assistance of Mr. W. K. Ossom, College of Agriculture and Renewable Natural Resources, KNUST and Mr. Degan Amissah of the KNUST Botanic Garden in the identification of plants.

\section{REFERENCES}

Begon, M., Harper, J.L. and Townsend, C.R. (1996). Ecology: Individuals, Populations, and Communities ( $3^{\text {rd }}$ edn.). Blackwell Science Ltd., Cambridge, MA.

Bhatt, Y.D., Rawat, Y.S. and Singh, S.P. (1994). Changes in ecosystem functioning after replacement of forest by Lantana shrubland in Kumaon Himalaya. Journal of Vegetation Science 5: 67-70.

Botanic Garden Conservation International (BGCI, 2005). http://www.bgci.org.org.uk/ botanic_garden/index.html. $5^{\text {th }}$ November, 2005.
Cox, G. W. (2002). General Ecology Laboratory Manual ( $8^{\text {th }}$ edn) McGraw-Hill Companies Inc. New York. 312 pp.

Hawthorne, W. (1990). Field Guide to the Forest Trees of Ghana. Chatham: Natural Resources Institute, for the Overseas Development Administration, London. Ghana Forestry Services 1: 278.

Hutchinson, J. and Dalziel, J. M. (1963). Flora of West Tropical Africa, Vol I (Parts 1 \& 2), Vol II. Crown Agency for Overseas Governments and Administration, Millbank, London.

Langenheim, J.H. and Thimann, K.V. (1982). Botany: Plant Biology and its Relation to Human Affairs. John Wiley and Sons Inc. USA. 624pp.

Munoz, A. A., Chacon, P., Perez, F., Barnert, E. S. and Armesto, J. J. (2003). Diversity and host species preference of vascular epiphytes and vines in a temperate rainforest in southern Chile. Australian Journal of Botany 51 (4): 381 - 391.

Pabst, R. J. and Spies, T. A. (1998). Distribution of herbs and shrubs in relation to landform and canopy cover in riparian forests of coastal Oregon. Can. J. Bot. 76: $298-315$.

Pennisi, E. (2005). What determines species diversity? Science 309 (5731): 90. DOI: 10.1126/science.309.5731.90

Richards, P. W. (1996). The tropical rainforest: an ecological study ( $2^{\text {nd }}$ edn). Cambridge University Press. 541pp.

Riffell, S. K. and Gutzwiller, K. J. (1996). Plant species richness in corridor intersections: is intersection shape influential? Landscape Ecology 11 (3): 157 - 168.

Smith, F.W., Long, J. N., and Vessey, G. (2004). The influence of canopy architecture on stemwood production and growth efficiency of Pinus contorta var. latifolia. Journal of Applied Ecology. 26: 681-691. 
Ssegawa, P. and Nkuutu, D.N. (2006). Diversity of vascular plants on Ssese Islands in Lake Victoria, Central Uganda. Afr. J. Ecol. 44: 22-29.

Tilman, D. (1988). Plant strategies and the $d y$ namics and structure of plant communities. Princeton University Press. Princeton, New Jersey.

van Andel, T. R. (2003). The use of distance measures in phytosociological sampling. Ecology 47: 451-460.

Watson, L. and Dallwitz, M. J. (2002). The families of flowering plants. http:// bodd.cf.ac.uk/BotDermFolder/BotDermC/ Comp.html. 1st December, 2005.

Willis, C. K. (2004). African Botanic Gardens Congress: Partnerships and Linkages. Proceedings of a Congress held at Durban Botanic Garden, South Africa 24 - 29 Novem- ber, 2002. Southern African Botanical Diversity Network Report No. 22. SABONET, Pretoria. 96pp

Willis, C. K., Botha, D. J. and Winter, J. H. S. (2002). Southern African botanic gardens need assessment. Southern African Botanical Diversity Network Report, 11. SABONET, Pretoria, South Africa. Pp. 344346.

Wyse-Jackson, P. S. and Sutherland, L. A. (2000). International agenda for botanic gardens in conservation. Botanic Gardens Conservation International, UK.

116 Journal of Science and Technology, Vol. 28, No. 3, Dec., 2008 
Appendix A

Distribution of Incomes of Households in Nkoranza District for 2000 and 2004 (in Ghana Cedis)

\begin{tabular}{|c|c|c|c|c|c|c|c|}
\hline \multicolumn{3}{|c|}{ Households A (HA) } & \multicolumn{3}{|c|}{ Households B (HB) } & \multirow{2}{*}{$\begin{array}{r}\text { HA } \\
\% \Delta \mathrm{HA}\end{array}$} & \multirow{2}{*}{$\begin{array}{c}\mathrm{HB} \\
\% \Delta \mathrm{HB}\end{array}$} \\
\hline Individual & $\begin{array}{c}\text { Income } \\
(2000)\end{array}$ & $\begin{array}{c}\text { Income } \\
(2004)\end{array}$ & Individual & $\begin{array}{c}\text { Income } \\
(2000)\end{array}$ & $\begin{array}{l}\text { Income } \\
(\mathbf{2 0 0 4})\end{array}$ & & \\
\hline HA 80 & 0.19 & 0.23 & HB50 & 0.20 & 0.25 & 21.05 & 25.00 \\
\hline HA 76 & 0.20 & 0.25 & HB75 & 0.21 & 0.30 & 25.00 & 42.86 \\
\hline HA64 & 0.20 & 0.30 & HB1 & 0.22 & 0.39 & 50.00 & 77.27 \\
\hline HA1 & 0.25 & 0.33 & HB55 & 0.27 & 0.39 & 32.00 & 44.44 \\
\hline HA10 & 0.28 & 0.40 & HB5 & 0.30 & 0.39 & 42.86 & 30.00 \\
\hline HA33 & 0.28 & 0.40 & HB11 & 0.30 & 0.40 & 42.86 & 33.33 \\
\hline HA53 & 0.30 & 0.40 & HB28 & 0.35 & 0.43 & 33.33 & 22.86 \\
\hline HA55 & 0.30 & 0.40 & HB94 & 0.36 & 0.45 & 33.33 & 25.00 \\
\hline HA45 & 0.30 & 0.42 & HB17 & 0.38 & 0.45 & 40.00 & 18.42 \\
\hline HA57 & 0.32 & 0.45 & HB24 & 0.39 & 0.48 & 40.63 & 23.08 \\
\hline HA2 & 0.32 & 0.47 & HB81 & 0.39 & 0.50 & 46.88 & 28.21 \\
\hline HA30 & 0.34 & 0.48 & HB20 & 0.40 & 0.50 & 41.18 & 25.00 \\
\hline HA43 & 0.35 & 0.48 & HB9 & 0.42 & 0.50 & 37.14 & 19.05 \\
\hline HA34 & 0.35 & 0.50 & HB13 & 0.45 & 0.55 & 42.86 & 22.22 \\
\hline HA36 & 0.37 & 0.50 & HB23 & 0.45 & 0.55 & 35.14 & 22.78 \\
\hline HA63 & 0.38 & 0.50 & HB63 & 0.47 & 0.56 & 31.58 & 19.15 \\
\hline HA44 & 0.38 & 0.51 & HB59 & 0.49 & 0.56 & 34.21 & 14.29 \\
\hline HA70 & 0.40 & 0.53 & HB10 & 0.50 & 0.58 & 32.50 & 16.00 \\
\hline HA37 & 0.40 & 0.55 & HB68 & 0.53 & 0.58 & 37.50 & 9.43 \\
\hline HA72 & 0.40 & 0.55 & HB37 & 0.58 & 0.60 & 37.50 & 3.45 \\
\hline HA9 & 0.42 & 0.60 & HB37 & 0.58 & 0.60 & 42.86 & 3.45 \\
\hline HA52 & 0.45 & 0.60 & HB12 & 0.59 & 0.60 & 33.33 & 1.69 \\
\hline HA58 & 0.45 & 0.60 & HB72 & 0.59 & 0.60 & 33.33 & 1.69 \\
\hline HA21 & 0.45 & 0.63 & HB52 & 0.60 & 0.60 & 40.00 & 0.00 \\
\hline HA68 & 0.45 & 0.64 & HB79 & 0.60 & 0.64 & 42.22 & 6.67 \\
\hline HA74 & 0.48 & 0.64 & HB88 & 0.60 & 0.65 & 33.33 & 8.33 \\
\hline HA8 & 0.50 & 0.65 & HB100 & 0.61 & 0.65 & 30.00 & 6.56 \\
\hline HA29 & 0.50 & 0.69 & HB53 & 0.62 & 0.65 & 38.00 & 4.84 \\
\hline HA46 & 0.50 & 0.69 & HB74 & 0.63 & 0.68 & 38.00 & 7.94 \\
\hline HA97 & 0.52 & 0.69 & HB84 & 0.63 & 0.68 & 32.69 & 7.94 \\
\hline HA82 & 0.52 & 0.70 & HB92 & 0.63 & 0.68 & 34.62 & 7.94 \\
\hline HA27 & 0.54 & 0.71 & HB32 & 0.65 & 0.68 & 31.48 & 4.62 \\
\hline HA40 & 0.54 & 0.71 & HB33 & 0.65 & 0.69 & 31.48 & 6.15 \\
\hline HA12 & 0.54 & 0.75 & HB56 & 0.68 & 0.69 & 38.89 & 1.47 \\
\hline HA86 & 0.54 & 0.75 & HB80 & 0.68 & 0.69 & 38.89 & 1.47 \\
\hline HA15 & 0.60 & 0.78 & HB18 & 0.69 & 0.70 & 30.00 & 1.45 \\
\hline
\end{tabular}


Floristic composition and vegetation structure of the KNUST ...

Anning et al.

\begin{tabular}{|c|c|c|c|c|c|c|c|}
\hline \multicolumn{3}{|c|}{ Households A (HA) } & \multicolumn{3}{|c|}{ Households B (HB) } & \multirow{2}{*}{$\begin{array}{r}\text { HA } \\
\% \Delta \mathrm{HA}\end{array}$} & \multirow{2}{*}{$\begin{array}{c}\text { HB } \\
\% \Delta \mathrm{HB}\end{array}$} \\
\hline Individual & $\begin{array}{c}\text { Income } \\
(2000)\end{array}$ & $\begin{array}{l}\text { Income } \\
(\mathbf{2 0 0 4})\end{array}$ & Individual & $\begin{array}{c}\text { Income } \\
(2000)\end{array}$ & $\begin{array}{c}\text { Income } \\
\text { (2004) }\end{array}$ & & \\
\hline HA28 & 0.60 & 0.78 & HB38 & 0.69 & 0.70 & 30.00 & 1.45 \\
\hline HA60 & 0.62 & 0.79 & HB60 & 0.69 & 0.70 & 27.42 & 1.45 \\
\hline HA5 & 0.64 & 0.80 & HB19 & 0.70 & 0.70 & 25.00 & 0.00 \\
\hline HA24 & 0.65 & 0.80 & HB43 & 0.70 & 0.70 & 23.08 & 0.00 \\
\hline HA83 & 0.65 & 0.80 & HB76 & 0.70 & 0.70 & 23.08 & 0.00 \\
\hline HA100 & 0.68 & 0.80 & HB78 & 0.70 & 0.70 & 17.65 & 0.00 \\
\hline HA13 & 0.70 & 0.83 & HB45 & 0.71 & 0.70 & 18.57 & -1.41 \\
\hline HA26 & 0.70 & 0.83 & HB26 & 0.73 & 0.71 & 18.57 & -2.74 \\
\hline HA48 & 0.70 & 0.83 & HB29 & 0.73 & 0.72 & 18.57 & -1.37 \\
\hline HA50 & 0.70 & 0.83 & HB71 & 0.74 & 0.73 & 18.57 & -1.35 \\
\hline HA41 & 0.72 & 0.85 & HB96 & 0.74 & 0.74 & 18.06 & 0.00 \\
\hline HA51 & 0.74 & 0.85 & HB22 & 0.75 & 0.75 & 14.86 & 0.00 \\
\hline HA85 & 0.74 & 0.85 & HB34 & 0.75 & 0.79 & 14.86 & 5.33 \\
\hline HA4 & 0.74 & 0.86 & HB47 & 0.75 & 0.79 & 16.22 & 5.33 \\
\hline HA54 & 0.75 & 0.86 & HB64 & 0.75 & 0.80 & 14.67 & 6.67 \\
\hline НА67 & 0.78 & 0.90 & HB82 & 0.75 & 0.80 & 15.38 & 6.67 \\
\hline HA79 & 0.78 & 0.90 & HB40 & 0.76 & 0.80 & 15.38 & 5.26 \\
\hline HA14 & 0.80 & 0.91 & HB41 & 0.78 & 0.80 & 13.75 & 2.56 \\
\hline HA49 & 0.80 & 0.91 & HB48 & 0.78 & 0.80 & 13.75 & 2.56 \\
\hline HA84 & 0.80 & 0.91 & HB62 & 0.78 & 0.82 & 13.75 & 5.13 \\
\hline HA3 & 0.84 & 0.92 & HB14 & 0.80 & 0.83 & 9.52 & 3.75 \\
\hline HA22 & 0.85 & 0.92 & HB30 & 0.80 & 0.83 & 8.24 & 3.75 \\
\hline HA32 & 0.85 & 0.92 & HB73 & 0.80 & 0.89 & 8.24 & 11.25 \\
\hline HA7 & 0.86 & 0.93 & HB97 & 0.80 & 0.90 & 8.14 & 12.50 \\
\hline HA31 & 0.88 & 0.93 & HB16 & 0.85 & 0.90 & 5.68 & 5.88 \\
\hline HA78 & 0.88 & 0.93 & HB27 & 0.89 & 0.90 & 5.68 & 1.12 \\
\hline HA99 & 0.90 & 0.94 & HB2 & 0.90 & 0.90 & 4.44 & 0.00 \\
\hline HA81 & 0.90 & 0.94 & HB4 & 0.90 & 0.90 & 4.44 & 0.00 \\
\hline HA11 & 0.90 & 0.94 & HB35 & 0.90 & 0.90 & 4.44 & 0.00 \\
\hline HA25 & 0.90 & 0.95 & HB51 & 0.90 & 0.90 & 5.56 & 0.00 \\
\hline HA18 & 0.90 & 0.95 & HB67 & 0.90 & 0.90 & 5.56 & 0.00 \\
\hline HA39 & 0.90 & 0.95 & HB86 & 0.90 & 0.90 & 5.56 & 0.00 \\
\hline HA47 & 0.90 & 0.95 & HB7 & 0.91 & 0.90 & 5.56 & -1.10 \\
\hline HA66 & 0.90 & 0.95 & HB57 & 0.91 & 0.91 & 5.56 & -0.55 \\
\hline HA95 & 0.90 & 0.96 & HB65 & 0.91 & 0.91 & 6.67 & -0.55 \\
\hline НА90 & 0.90 & 0.96 & HB42 & 0.92 & 0.91 & 6.67 & -1.09 \\
\hline HA20 & 0.90 & 0.98 & HB49 & 0.92 & 0.92 & 8.89 & 0.00 \\
\hline HA16 & 0.90 & 1.00 & HB66 & 0.92 & 0.93 & 11.11 & 0.54 \\
\hline HA73 & 0.91 & 1.00 & HB85 & 0.92 & 0.93 & 9.89 & 1.09 \\
\hline
\end{tabular}

118 Journal of Science and Technology, Vol. 28, No. 3, Dec., 2008 
Floristic composition and vegetation structure of the KNUST ...

Anning et al.

\begin{tabular}{|c|c|c|c|c|c|c|c|}
\hline \multicolumn{3}{|c|}{ Households A (HA) } & \multicolumn{3}{|c|}{ Households B (HB) } & \multirow{2}{*}{$\begin{array}{r}\text { HА } \\
\% \Delta \mathrm{HA}\end{array}$} & \multirow{2}{*}{$\begin{array}{c}\mathrm{HB} \\
\% \Delta \mathrm{HB}\end{array}$} \\
\hline Individual & $\begin{array}{c}\text { Income } \\
(2000)\end{array}$ & $\begin{array}{c}\text { Income } \\
(2004)\end{array}$ & Individual & $\begin{array}{c}\text { Income } \\
(2000)\end{array}$ & $\begin{array}{c}\text { Income } \\
(2004)\end{array}$ & & \\
\hline HA96 & 0.92 & 1.00 & HB95 & 0.92 & 0.93 & 8.70 & 1.09 \\
\hline HА93 & 0.92 & 1.20 & HB98 & 0.92 & 0.94 & 30.43 & 2.17 \\
\hline HA38 & 0.92 & 1.20 & HB36 & 0.93 & 0.94 & 30.43 & 1.08 \\
\hline HA6 & 0.94 & 1.20 & HB77 & 0.94 & 0.95 & 27.66 & 0.53 \\
\hline HA56 & 0.95 & 1.20 & HB93 & 0.95 & 0.95 & 26.32 & 0.53 \\
\hline HA98 & 0.95 & 1.35 & HB70 & 0.95 & 0.95 & 42.11 & 0.00 \\
\hline HA91 & 0.95 & 1.35 & HB6 & 0.95 & 0.96 & 42.11 & 1.05 \\
\hline HA35 & 0.95 & 1.40 & HB25 & 0.96 & 0.96 & 47.37 & 0.00 \\
\hline HA17 & 0.98 & 1.40 & HB44 & 0.96 & 0.96 & 42.86 & 0.00 \\
\hline HA71 & 1.00 & 1.40 & HB99 & 0.97 & 0.97 & 40.00 & 0.52 \\
\hline HA87 & 1.00 & 1.40 & HB69 & 0.97 & 0.97 & 40.00 & 0.00 \\
\hline HA59 & 1.00 & 1.40 & HB83 & 0.99 & 0.98 & 40.00 & -0.51 \\
\hline HA19 & 1.10 & 1.50 & HB39 & 1.00 & 1.00 & 36.36 & 0.00 \\
\hline HA23 & 1.20 & 1.50 & НB90 & 1.00 & 1.10 & 25.00 & 10.00 \\
\hline HA42 & 1.20 & 1.50 & HB87 & 1.20 & 1.15 & 25.00 & -4.17 \\
\hline HA75 & 1.20 & 1.50 & HB15 & 1.20 & 1.20 & 25.00 & 0.00 \\
\hline HA69 & 1.20 & 1.80 & HB90 & 1.30 & 1.25 & 50.00 & -3.85 \\
\hline HA65 & 1.30 & 1.80 & HB31 & 1.30 & 1.25 & 38.46 & -3.85 \\
\hline HA94 & 1.50 & 1.80 & HB58 & 1.30 & 1.30 & 20.00 & 0.00 \\
\hline HA61 & 1.50 & 2.00 & HB91 & 1.30 & 1.30 & 33.33 & 0.00 \\
\hline HA88 & 1.50 & 2.00 & HB61 & 1.30 & 1.35 & 33.33 & 3.85 \\
\hline HA62 & 1.50 & 2.20 & HB46 & 1.40 & 1.41 & 46.67 & 0.71 \\
\hline HA89 & 1.80 & 2.50 & HB21 & 1.42 & 1.55 & 38.89 & 9.15 \\
\hline HA92 & 1.80 & 2.60 & HB54 & 1.50 & 1.70 & 44.44 & 13.33 \\
\hline HA77 & 2.00 & 2.60 & HB8 & 1.65 & 1.80 & 30.00 & 9.09 \\
\hline
\end{tabular}

Source: Field Survey, 2004 
Appendix B

Distribution of Incomes of Households in Wenchi District for 2000 and 2004 (in Ghana Cedis)

\begin{tabular}{|c|c|c|c|c|c|c|c|}
\hline \multicolumn{3}{|c|}{ Households A (HA) } & \multicolumn{3}{|c|}{ Households B (HB) } & \multirow{2}{*}{$\begin{array}{c}\text { HA } \\
\% \Delta H A\end{array}$} & \multirow{2}{*}{$\begin{array}{c}\text { HB } \\
\% \Delta \mathrm{HA}\end{array}$} \\
\hline Individual & $\begin{array}{c}\text { Income } \\
(2000)\end{array}$ & $\begin{array}{c}\text { Income } \\
(2004)\end{array}$ & Individual & $\begin{array}{c}\text { Income } \\
(2000)\end{array}$ & $\begin{array}{c}\text { Income } \\
(2004)\end{array}$ & & \\
\hline HA56 & 0.19 & 0.20 & HB84 & 0.19 & 0.20 & 5.26 & 5.26 \\
\hline HA79 & 0.20 & 0.20 & HB12 & 0.21 & 0.20 & 2.56 & -4.76 \\
\hline HA74 & 0.20 & 0.33 & HB24 & 0.30 & 0.30 & 65.00 & 0.00 \\
\hline HA81 & 0.22 & 0.33 & HB43 & 0.35 & 0.32 & 50.00 & -8.57 \\
\hline HА92 & 0.23 & 0.34 & HB82 & 0.36 & 0.36 & 47.83 & 0.00 \\
\hline HA71 & 0.25 & 0.40 & HB81 & 0.37 & 0.38 & 60.00 & 2.70 \\
\hline HA72 & 0.26 & 0.41 & HB22 & 0.40 & 0.41 & 57.69 & 2.50 \\
\hline HA95 & 0.30 & 0.41 & HB2 & 0.43 & 0.42 & 36.67 & -2.33 \\
\hline HA91 & 0.32 & 0.41 & HB31 & 0.45 & 0.45 & 28.75 & 0.00 \\
\hline HA99 & 0.32 & 0.43 & HB66 & 0.48 & 0.49 & 34.38 & 2.08 \\
\hline HA83 & 0.33 & 0.48 & HB68 & 0.49 & 0.50 & 45.45 & 2.04 \\
\hline HA76 & 0.34 & 0.50 & HB80 & 0.50 & 0.50 & 47.06 & 0.00 \\
\hline HA97 & 0.35 & 0.52 & HB88 & 0.50 & 0.52 & 48.57 & 4.00 \\
\hline HA23 & 0.36 & 0.53 & HB79 & 0.55 & 0.55 & 47.22 & 0.00 \\
\hline НА69 & 0.37 & 0.53 & HB1 & 0.55 & 0.55 & 43.24 & -0.45 \\
\hline HA49 & 0.38 & 0.56 & HB30 & 0.56 & 0.57 & 47.37 & 1.79 \\
\hline HA51 & 0.39 & 0.58 & HB87 & 0.56 & 0.57 & 48.72 & 1.79 \\
\hline HA50 & 0.40 & 0.59 & HB18 & 0.58 & 0.58 & 47.50 & 0.00 \\
\hline HA13 & 0.41 & 0.60 & HB42 & 0.58 & 0.59 & 46.34 & 1.72 \\
\hline HA31 & 0.42 & 0.60 & HB35 & 0.60 & 0.61 & 42.86 & 1.67 \\
\hline HA60 & 0.42 & 0.60 & HB41 & 0.60 & 0.62 & 42.86 & 3.33 \\
\hline HA78 & 0.43 & 0.60 & HB50 & 0.60 & 0.62 & 39.53 & 3.33 \\
\hline HA93 & 0.44 & 0.64 & HB58 & 0.60 & 0.63 & 45.45 & 5.00 \\
\hline HA34 & 0.45 & 0.65 & HB99 & 0.60 & 0.63 & 44.44 & 5.00 \\
\hline НА66 & 0.47 & 0.65 & HB65 & 0.64 & 0.65 & 38.30 & 1.56 \\
\hline HA82 & 0.48 & 0.65 & HB75 & 0.65 & 0.65 & 35.42 & 0.00 \\
\hline HA94 & 0.50 & 0.65 & HB77 & 0.65 & 0.66 & 30.00 & 1.54 \\
\hline HA19 & 0.51 & 0.67 & HB90 & 0.65 & 0.67 & 31.37 & 3.08 \\
\hline HA20 & 0.51 & 0.68 & HB20 & 0.68 & 0.69 & 33.33 & 1.47 \\
\hline HA10 & 0.52 & 0.69 & HB23 & 0.68 & 0.68 & 32.69 & 0.00 \\
\hline HA45 & 0.52 & 0.69 & HB64 & 0.68 & 0.69 & 32.69 & 1.47 \\
\hline HA90 & 0.53 & 0.69 & HB71 & 0.68 & 0.69 & 30.19 & 1.47 \\
\hline HA22 & 0.54 & 0.70 & HB47 & 0.69 & 0.69 & 29.63 & 0.00 \\
\hline HA35 & 0.55 & 0.70 & HB89 & 0.69 & 0.70 & 27.27 & 1.45 \\
\hline HA36 & 0.56 & 0.70 & HB93 & 0.68 & 0.71 & 25.00 & 4.41 \\
\hline
\end{tabular}

120 Journal of Science and Technology, Vol. 28, No. 3, Dec., 2008 
Floristic composition and vegetation structure of the KNUST ...

Anning et al.

\begin{tabular}{|c|c|c|c|c|c|c|c|}
\hline \multicolumn{3}{|c|}{ Households A (HA) } & \multicolumn{3}{|c|}{ Households B (HB) } & \multirow{2}{*}{$\begin{array}{c}\text { HA } \\
\% \Delta \mathbf{H A}\end{array}$} & \multirow{2}{*}{$\begin{array}{c}\text { HB } \\
\% \Delta \mathrm{HA}\end{array}$} \\
\hline Individual & $\begin{array}{c}\text { Income } \\
\text { (2000) }\end{array}$ & $\begin{array}{c}\text { Income } \\
\text { (2004) }\end{array}$ & Individual & $\begin{array}{c}\text { Income } \\
(2000)\end{array}$ & $\begin{array}{c}\text { Income } \\
\text { (2004) }\end{array}$ & & \\
\hline HA55 & 0.58 & 0.70 & HB19 & 0.70 & 0.72 & 20.69 & 2.14 \\
\hline HA42 & 0.59 & 0.71 & HB34 & 0.70 & 0.72 & 20.34 & 2.14 \\
\hline HA43 & 0.60 & 0.74 & HB39 & 0.70 & 0.72 & 23.33 & 2.86 \\
\hline HA6 & 0.61 & 0.75 & HB48 & 0.70 & 0.72 & 22.95 & 2.86 \\
\hline HA25 & 0.63 & 0.75 & HB52 & 0.70 & 0.72 & 19.05 & 2.86 \\
\hline HA84 & 0.65 & 0.78 & HB53 & 0.70 & 0.73 & 20.00 & 4.29 \\
\hline HA30 & 0.67 & 0.80 & HB85 & 0.70 & 0.73 & 19.40 & 4.29 \\
\hline HA61 & 0.69 & 0.80 & HB91 & 0.70 & 0.73 & 15.94 & 4.29 \\
\hline HA86 & 0.70 & 0.81 & HB70 & 0.71 & 0.73 & 15.71 & 2.82 \\
\hline HA48 & 0.71 & 0.82 & HB6 & 0.72 & 0.74 & 15.49 & 2.78 \\
\hline HA67 & 0.71 & 0.83 & HB73 & 0.73 & 0.74 & 16.90 & 1.37 \\
\hline HA17 & 0.72 & 0.85 & HB10 & 0.74 & 0.75 & 18.06 & 1.35 \\
\hline HA8 & 0.73 & 0.90 & HB29 & 0.75 & 0.75 & 23.29 & 0.00 \\
\hline HA18 & 0.74 & 0.90 & HB78 & 0.79 & 0.76 & 21.62 & -3.80 \\
\hline HA21 & 0.75 & 0.90 & HB97 & 0.79 & 0.77 & 20.00 & -2.53 \\
\hline HA37 & 0.75 & 0.90 & HB7 & 0.80 & 0.78 & 20.00 & -2.50 \\
\hline HA46 & 0.76 & 0.90 & HB8 & 0.80 & 0.79 & 18.42 & -1.25 \\
\hline HA53 & 0.77 & 0.90 & HB14 & 0.80 & 0.81 & 16.88 & 1.25 \\
\hline HA68 & 0.79 & 0.90 & HB27 & 0.80 & 0.82 & 13.92 & 2.50 \\
\hline HA98 & 0.80 & 0.90 & HB96 & 0.80 & 0.83 & 12.50 & 3.75 \\
\hline HA2 & 0.82 & 0.91 & HB32 & 0.82 & 0.83 & 10.98 & 1.22 \\
\hline HA85 & 0.83 & 0.91 & HB26 & 0.83 & 0.84 & 9.64 & 1.20 \\
\hline HA89 & 0.84 & 0.91 & HB67 & 0.83 & 0.85 & 8.33 & 2.41 \\
\hline HA14 & 0.85 & 0.92 & HB83 & 0.89 & 0.88 & 8.24 & -1.12 \\
\hline HA73 & 0.86 & 0.92 & HB21 & 0.90 & 0.91 & 6.98 & 1.11 \\
\hline HA4 & 0.87 & 0.93 & HB25 & 0.90 & 0.91 & 6.90 & 1.11 \\
\hline HA9 & 0.88 & 0.93 & HB40 & 0.90 & 0.92 & 5.68 & 2.22 \\
\hline HA70 & 0.89 & 0.93 & HB55 & 0.90 & 0.92 & 4.49 & 2.22 \\
\hline HA100 & 0.90 & 0.94 & HB56 & 0.90 & 0.92 & 3.89 & 2.22 \\
\hline HA1 & 0.90 & 0.94 & HB72 & 0.90 & 0.93 & 4.44 & 3.33 \\
\hline HA26 & 0.91 & 0.94 & HB74 & 0.90 & 0.91 & 3.30 & 1.11 \\
\hline HA7 & 0.91 & 0.95 & HB86 & 0.90 & 0.92 & 4.40 & 2.22 \\
\hline HA38 & 0.92 & 0.95 & HB95 & 0.90 & 0.92 & 3.26 & 2.22 \\
\hline HA63 & 0.92 & 0.96 & HB98 & 0.90 & 0.93 & 4.35 & 3.33 \\
\hline HA65 & 0.92 & 0.96 & HB9 & 0.91 & 0.94 & 4.35 & 3.31 \\
\hline HA88 & 0.93 & 0.97 & HB44 & 0.91 & 0.94 & 4.30 & 3.31 \\
\hline HA75 & 0.93 & 0.98 & HB28 & 0.91 & 0.93 & 5.38 & 2.20 \\
\hline HA96 & 0.93 & 0.99 & HB37 & 0.91 & 0.94 & 6.45 & 2.75 \\
\hline HA3 & 0.93 & 1.00 & HB51 & 0.91 & 0.94 & 7.53 & 2.75 \\
\hline HA15 & 0.93 & 1.01 & HB59 & 0.91 & 0.93 & 8.60 & 2.20 \\
\hline
\end{tabular}

Journal of Science and Technology, Vol. 28, No. 3, Dec., 2008121 
Floristic composition and vegetation structure of the KNUST ...

Anning et al.

\begin{tabular}{|c|c|c|c|c|c|c|c|}
\hline \multicolumn{3}{|c|}{ Households A (HA) } & \multicolumn{3}{|c|}{ Households B (HB) } & \multirow{2}{*}{$\begin{array}{c}\text { HA } \\
\% \Delta H A\end{array}$} & \multirow{2}{*}{$\begin{array}{c}\text { HB } \\
\% \Delta \mathrm{HA}\end{array}$} \\
\hline Individual & $\begin{array}{c}\text { Income } \\
(\mathbf{2 0 0 0 )}\end{array}$ & $\begin{array}{c}\text { Income } \\
\text { (2004) }\end{array}$ & Individual & $\begin{array}{c}\text { Income } \\
(2000)\end{array}$ & $\begin{array}{c}\text { Income } \\
\text { (2004) }\end{array}$ & & \\
\hline HA57 & 0.93 & 1.03 & HB17 & 0.92 & 0.94 & 10.75 & 2.17 \\
\hline HA52 & 0.94 & 1.05 & HB36 & 0.92 & 0.95 & 12.30 & 3.26 \\
\hline HA77 & 0.94 & 1.08 & HB54 & 0.92 & 0.96 & 15.51 & 4.35 \\
\hline HA59 & 0.94 & 1.20 & HB100 & 0.93 & 0.97 & 28.34 & 3.76 \\
\hline HA5 & 0.94 & 1.22 & HB4 & 0.94 & 0.97 & 29.79 & 3.19 \\
\hline HA16 & 0.95 & 1.25 & HB13 & 0.95 & 0.98 & 31.58 & 3.16 \\
\hline HA11 & 0.96 & 1.26 & HB76 & 0.96 & 0.99 & 31.25 & 3.66 \\
\hline HA40 & 0.99 & 1.28 & HB92 & 0.96 & 0.99 & 29.29 & 3.13 \\
\hline HA39 & 1.00 & 1.28 & HB69 & 0.96 & 1.00 & 28.00 & 4.17 \\
\hline HA29 & 1.01 & 1.30 & HB94 & 0.97 & 1.10 & 28.71 & 13.40 \\
\hline HA33 & 1.02 & 1.35 & HB57 & 0.97 & 1.15 & 32.35 & 18.56 \\
\hline HA80 & 1.03 & 1.36 & HB3 & 0.98 & 1.20 & 32.04 & 22.45 \\
\hline HA62 & 1.05 & 1.39 & HB33 & 0.99 & 1.25 & 32.38 & 26.26 \\
\hline HA28 & 1.10 & 1.40 & HB62 & 1.00 & 1.27 & 27.27 & 27.00 \\
\hline HA32 & 1.12 & 1.45 & HB60 & 1.10 & 1.28 & 29.46 & 16.36 \\
\hline HA87 & 1.16 & 1.50 & HB46 & 1.20 & 1.30 & 29.31 & 8.33 \\
\hline HA41 & 1.19 & 1.52 & HB63 & 1.30 & 1.35 & 27.73 & 3.85 \\
\hline HA27 & 1.20 & 1.56 & HB61 & 1.30 & 1.38 & 30.00 & 6.15 \\
\hline HA24 & 1.25 & 1.56 & HB15 & 1.40 & 1.40 & 24.80 & 0.00 \\
\hline HA12 & 1.30 & 1.66 & HB11 & 1.40 & 1.45 & 27.69 & 3.57 \\
\hline HA47 & 1.35 & 1.70 & HB5 & 1.45 & 1.45 & 25.93 & 0.00 \\
\hline HA64 & 1.38 & 1.78 & HB38 & 1.45 & 1.48 & 28.99 & 2.07 \\
\hline HA54 & 1.40 & 1.80 & HB49 & 1.50 & 1.55 & 28.57 & 3.33 \\
\hline HA58 & 1.50 & 1.85 & HB16 & 1.60 & 1.80 & 23.33 & 12.50 \\
\hline HA44 & 1.60 & 2.20 & HB45 & 1.70 & 1.90 & 37.50 & 11.76 \\
\hline
\end{tabular}

Source: Field Survey, 2004

122 Journal of Science and Technology, Vol. 28, No. 3, Dec., 2008 